\title{
ESTADOS UNIDOS FRENTE A LA PROMULGACIÓN Y SUSPENSIÓN EN MÉXICO DEL REGLAMENTO DE TOXICOMANÍAS DE 1940
}

\author{
THE UNITED STATES AND THE PROMULGATION AND \\ SUSPENSION IN MEXICO OF THE 1940 \\ REGULATION OF DRUG ADDICTION
}

\section{LES ÉTATS-UNIS FACE À LA PROMULGATION ET LA SUSPENSION AU MEXIQUE DU RĖGLEMENT DE 1940 SUR LES TOXICOMANIES}

\author{
Carlos A. Pérez Ricart \\ División de Estudios Internacionales, \\ Centro de Investigación y \\ Docencia Económicas (CIDE) \\ cperezricart@gmail.com
}

Resumen: Este artículo analiza el proceso de promulgación y suspensión en México del Reglamento de Toxicomanías de 1940, por el que se permitía, entre otras cosas, la puesta en marcha de un programa de sustitución de adicciones radical para su época. Aunque el Reglamento fue firmado por el presidente Lázaro Cárdenas en enero, en junio de 1940 fue suspendido de forma indefinida debido a presiones de diferentes instituciones del gobierno de Estados Unidos (EE.UU.).

$\mathrm{El}$ artículo tiene dos objetivos. Por un lado, examinar el papel que desempeñaron diferentes actores e instituciones del gobierno de EE.UU. en la suspensión del referido Reglamento. Por otro, analizar la reacción tanto de la Secretaría de Relaciones Exteriores (SRE) como del Departamento de Salubridad Pública (DSP) en defensa del mismo.

Abrevando de fuentes primarias reunidas en México y en EE.uU., se reconstruyen los pormenores de la relación transgubernamental entre funcionarios mexicanos y estadounidenses entre abril de 1938 y julio de 1940, mes en el que se suspende el Reglamento.

Palabras clave: política de drogas; reglamento de toxicomanías; relaciones transgubernamentales; México-Estados Unidos;

Leopoldo Salazar Viniegra. 
Abstract: This article analyzes the process behind the enactment and subsequent suspension in Mexico of the Federal Regulation on Drug Addiction 1940, which permitted among other things the implementation of an addiction substitution program that was radical for its time. Although this Regulation was signed by President Lázaro Cárdenas in January, by June 1940 it was suspended indefinitely due to pressure from U.S. government agencies.

The paper has two goals. On the one hand, to examine the role played by different actors and institutions of the U.S. government in the suspension of this Regulation. On the other, to analyze the reaction of both the Ministry of Foreign Affairs (SRE) and the Department of Public Health (DSP) to defend it.

Drawing on primary sources gathered in Mexico and in the U.S., the details of the cross-governmental relationship between Mexican and U.S. offcials between April 1938 and July 1940, the month in which the Regulation was suspended.

Keywords: drugs policy; drug addiction regulations; cross-governmental relationships; Mexico-United States; Leopoldo Salazar Viniegra.

\section{Traducción de Fionn Petch, CM IDiomas}

Résumé: Cet article analyse le processus de promulgation et de suspension au Mexique du Règlement sur la toxicomanie de 1940, qui a permis, entre autres, la mise en œuvre d'un programme de substitution des toxicomanies radical pour l'époque. Bien que le règlement ait été signé par le président Lázaro Cárdenas en janvier, en juin 1940, il a été suspendu indéfiniment en raison des pressions exercées par différentes institutions du gouvernement des États-Unis.

L'article a deux objectifs. D'une part, examiner le rôle joué par les différents acteurs et institutions du gouvernement américain dans la suspension du règlement précité. De l'autre, analyser la réaction du Ministère des relations extérieures (SRE) et de la Direction de la santé publique (DSP) pour défendre celui-ci.

S'appuyant sur des sources primaires rassemblées au Mexique et aux États-Unis, sont reconstitués les détails de la relation transgouvernementale entre les responsables mexicains et américains entre avril 1938 et juillet 1940, mois où le règlement est suspendu. 

Mots clés: politique pharmaceutique; réglementation pharmaceutique ; relations transgouvernementales ; Mexique-États-Unis ; Leopoldo Salazar Viniegra.

Traducción de Rafael Segovia, CM Idiomas

Fecha de recepción: junio de 2020

Fecha de aceptación: abril de 2021

\section{EL CONTEXTO}

$\mathrm{L}$ a mañana del sábado 9 de marzo de 1940 era fría pero, aun así, la Banda de Música del Estado Mayor entonaba piezas alegres. Los camarógrafos y periodistas se amotinaban en las afueras del edificio situado en el número 33 de la calle Sevilla de la colonia Juárez y buscaban la mejor imagen de los protagonistas de la noticia: los médicos que celebraban la apertura del primer dispensario de morfina para los -según el lenguaje de la época-toxicómanos en la Ciudad de México. ${ }^{1}$ Aquel dispensario, el primero de muchos planeados para todo el país, era la punta de lanza de un amplio programa que suponía, entre otras iniciativas, la creación de un monopolio estatal que surtiría morfina, a precios nominales y previamente recetada por médicos, a los toxicómanos.

El objetivo era aliviar la demanda de morfina de los adictos. En el mejor de los casos, explicaban los médicos aquella mañana, se lograría reducir progresivamente la dosis requerida y así iniciar un proceso de desintoxicación paulatina.

${ }^{1}$ Para una descripción de lo acontecido aquella mañana: Ricardo Pérez Montfort, Tolerancia y prohibición: aproximaciones a la historia social y cultural de las drogas en México (1840-1940), México, D.F., Debate, 2016, p. 296. En este texto utilizaré los términos "toxicómanos" y "adictos" en cursiva por tratarse de categorías socialmente construidas y cuya definición ha ido cambiando de acuerdo a convenciones sociales específicas. 
Así, el adicto dispuesto a entrar en el programa, podría continuar su vida normal si el médico en cuestión no percibía que pudiera infringir algún daño a la sociedad. En el peor de los casos, aun cuando el toxicómano no pudiera curarse, la creación de dispensarios de morfina permitiría que éstos dejaran de depender de la droga ofrecida en el mercado ilegal. De esta manera, se reducirían las ganancias de las redes de narcotráfico que, ya por entonces, comenzaban a proliferar en el país. ${ }^{2}$ Además, al ofrecer un lugar seguro para inyectarse la morfina, con el dispensario se prometía reducir desde el contagio de enfermedades venéreas hasta el eventual comportamiento violento del toxicómano producido por la necesidad de conseguir dinero con el cual pagar su consumo.

Este programa, que hoy la bibliografía médica etiquetaría como de "mantenimiento de adicciones", era nuevo en México y su implementación se debía, en gran parte, al esfuerzo del médico duranguense Leopoldo Salazar Viniegra, otrora jefe de la Dirección de Toxicomanías e Higiene Mental (DTHM) del Departamento de Salubridad Pública (DSP) entre principios de 1938 y agosto de 1939. El programa, aunque inédito y tremendamente original en el contexto mexicano, era muy similar a otros planteados en otros países. ${ }^{3}$ En varias ciudades de Estados Unidos (EE.UU.), a finales de la década de 1910, se habían fundado docenas de clínicas y dispensarios que ofrecían morfina para toxicómanos a bajos precios, así como esquemas de mantenimiento de adicciones radicales para su época. ${ }^{4}$

${ }^{2}$ Véanse, por ejemplo: Nicole Mottier, "Drug Gangs and Politics in Ciudad Juárez: 1928-1936", Mexican Studies/Estudios Mexicanos 25, núm. 1 (2009), pp. 19-46; Luis Astorga, El siglo de las drogas: el narcotráfico, del porfiriato al nuevo milenio, México, Grijalbo/Proceso, 2012.

${ }^{3}$ Sobre este punto, véase Nathaniel Lee Smith, "“Cured of the Habit by Force': The United States and the Global Campaign to Punish Drug Consumers, 1898-1970", tesis de doctorado, Ann Arbor, The University of North Carolina at Chapel Hill, 2007, p. 180.

${ }^{4}$ Representative of the United States of America to the Advisory Committee on Traffic in Opium and other Dangerous Drugs, "Experien- 
Durante su breve periodo como jefe de la DTMH, Salazar había conseguido convencer a su jefe directo, Leónides Andreu Almazán, de apoyar sus esfuerzos para implementar un programa de este calibre en México. El vehículo que había encontrado Salazar para ello era la reforma del Reglamento Federal de Toxicomanías cuya versión de 1931 regulaba el tratamiento de adictos y mandataba la reclusión hospitalaria de todo toxicómano. ${ }^{5}$

Tras una amplia negociación entre diferentes actores e instituciones relacionadas con el campo de la salud en México -que incluyó al propio Departamento Jurídico del DSP, "baluarte o abismo donde se estrellan o se hunden generalmente todas las iniciativas de provecho"6-, Salazar impulsó, durante su corto periodo al frente de la DTMH, la reforma al Reglamento de 1931, misma que fue firmada por el presidente Lázaro Cárdenas el 5 de enero de 1940. El 17 de febrero apareció publicada en el Diario Oficial de la Federación (DOF). Tres semanas después, la Banda de Música del Estado mayor amenizaba la apertura del dispensario.

En la reforma del Reglamento de 1940 se anunciaba la intención del DSP de construir y adecuar los hospitales y dispensarios necesarios. Estos -dictaba el artículo octavo- llevarían registros de los tratamientos de los pacientes, despacharían las sustancias narcóticas bajo estrictos procedimientos y administrarían "al toxicómano la droga que le haya sido prescrita, cuando éste la pida y mediante el pago de su importe". ${ }^{7}$ En resumen: la reforma renegaba del "sistema de persecución y denuncia de los toxicómanos" por ser "contrario al concepto

ce in the United States of America with the plan of selling drugs to addicts at low prices”, 22 de octubre de 1935, RG 59 812.114, NARA.

5 "El Reglamento Federal de Toxicomanías y los compromisos contraídos por México en las Convenciones Internacionales vigentes", 26 de marzo de 1940, Topografía III -2398-6, Archivo Histórico Genaro Estrada.

${ }^{6}$ Leopoldo Salazar Viniegra, "Opio y política: historia de una humillación”, Excélsior, 19 de diciembre de 1945.

${ }^{7}$ Reglamento Federal de Toxicomanías del Departamento de Salubridad Pública, 1940, Diario Oficial de la Federación, 17 de febrero de 1940. 
de justicia" y promovía una conceptualización del "vicioso más como enfermo a quien hay que atender y curar, que como verdadero delincuente que debe sufrir una pena". ${ }^{8}$ En definitiva, "al establecer la libertad de tratamiento, la facultad de recetar dosis de enervantes superiores a las señaladas en la farmacopea y al abrir al público expendios oficiales y particulares de drogas, fija [ba] un sistema, radicalmente distinto al que se venía siguiendo". ${ }^{9}$

El dispensario de la calle Sevilla funcionó durante algunas semanas. Sin embargo, antes de que pudieran analizarse sus virtudes o potenciales problemas, de manera sorpresiva para el público, el tres de julio de 1940, el Dof publicó “por tiempo indefinido" la suspensión del Reglamento de Toxicomanías apenas promulgado. La población no lo sabía, pero aquel experimento había terminado como consecuencia de la presión, tras bambalinas, que durante meses había ejercido sobre el DSP y la Secretaría de Relaciones Exteriores (SRE) el gobierno de EE.UU., en particular, la Oficina de Narcóticos (Federal Bureau of Narcotics, FBN), el Departamento de Estado y la Oficina de Aduanas de ese país. ¿Por qué canales transcurrió esa presión? ¿Cómo fue el proceso de negociación? ¿Bajo qué términos cedió el gobierno mexicano a la presión estadounidense? Ésas son preguntas que aborda el presente artículo.

\section{ObJetivos DEL ARTíCulo y ESTAdo de LA CUESTIÓN}

Este texto tiene dos objetivos. Por un lado, examinar el papel que desempeñaron diferentes actores e instituciones del gobierno de EE.uU. en la suspensión del referido Reglamento de Toxicomanías. Por otro lado, analizar la reacción tanto de

8 “Campaña contra una lacra social”, El Universal (sec. Editorial), 23 de mayo de 1940.

9 "El Reglamento Federal de Toxicomanías y los compromisos contraídos por México en las Convenciones Internacionales vigentes”. 
la SRE como del DSP en la defensa del plan de mantenimiento de adicciones.

Para lograr estos objetivos, el artículo reconstruye los pormenores de la relación transgubernamental entre funcionarios mexicanos y estadounidenses entre abril de 1938 -fecha en que la Oficina de Aduanas de EE.uU. se enteró de los planes de Salazar- y julio de 1940, mes en el que se suspende el Reglamento. ${ }^{10}$

El argumento central del artículo se desdobla en dos partes. En relación con el papel de EE.UU., el artículo sostiene que, a pesar de las diferencias e interpretaciones distintas sobre cómo abordar el problema, las principales instituciones y actores estadounidenses actuaron de manera consensuada. En lo esencial hubo anuencia en que sólo mediante la implementación de mecanismos de coerción -incluyendo la ejecución de un embargo medicinal- iba a lograr contenerse el plan de Salazar. Sin embargo, como se revisará, la estrategia estadounidense incluyó otros mecanismos mucho más sutiles que complementaron las medidas coercitivas y obtuvieron el efecto deseado: la suspensión del Reglamento.

En lo que cabe a la posición mexicana, el artículo mantiene que lo que verdaderamente caracterizó a los diplomáticos y a los responsables mexicanos de tomar las decisiones fue la falta de coordinación y, sobre todo, convencimiento de que la defensa del Reglamento era un tema suficientemente relevante como para forzar una disputa bilateral. Una combinación de varios factores que incluyen el desconocimiento de los postulados centrales de la reforma de 1940, el franco desinterés y problemas estructurales de comunicación entre dependencias, evitaron la defensa adecuada del

${ }^{10}$ Definimos relaciones transgubernamentales como el conjunto de vínculos, contactos e interacciones establecidos entre personajes u organizaciones subestatales (es decir, no centrales) de al menos dos Estados distintos. Véase J.S. Nye y R.O. Keohane, "Transgovernmental Relations and International Organizations", World Politics: A Quarterly Journal of International Relations, 1974, pp. 39-62. 
Reglamento en foros internacionales y en mesas de negociación con funcionarios estadounidenses.

El artículo busca contribuir a la investigación que ya han comenzado otros académicos sobre este momento crítico de la historia de la política antidrogas en México. ${ }^{11}$ Sin embargo, a diferencia de la mayoría de los trabajos sobre este tema, el enfoque está puesto menos en el contenido del Reglamento y más en el proceso de negociación entre instituciones mexicanas y estadounidenses entre 1938 y 1940. Esto es, en las relaciones transgubernamentales.

En relación con el tema específico del proceso de negociación, el artículo dialoga con tres textos que ya han analizado este momento, dos de reciente publicación y otro algo más antiguo. Todos ellos, sin embargo, han hecho contribuciones importantes para entender este periodo.

Isaac Campos fue el primero en examinar a profundidad la parte mexicana de la negociación. ${ }^{12}$ Según el análisis de Campos, quien basa su investigación en actas del gobierno mexicano otrora inadvertidas -las Actas de Sesión del Consejo de Salubridad-, en la reacción mexicana para enfrentar las presiones estadounidenses predominó la desorganiza-

${ }^{11}$ Isaac Campos, "A Diplomatic Failure: The Mexican Role in the Demise of the 1940 Reglamento Federal de Toxicomanías", Third World Quarterly 39, núm. 3 (2017); Pérez Montfort, Tolerancia y prohibición: aproximaciones a la historia social y cultural de las drogas en México (1840-1940); Mariana Flores Guevara, "La alternativa mexicana al marco internacional de prohibición de drogas durante el cardenismo", tesis de licenciatura, México, D.F., El Colegio de México, 2013; Benjamin T. Smith, "The Dialectics of Dope: Leopoldo Salazar Viniegra, the Myth of Marijuana, and Mexico's State Drug Monopoly", en Susannah Wilson (ed.), Prohibitions and Psychoactive Substances in History, Culture and Theory, Londres, Routledge, 2019, pp. 111-32; Froylán Enciso, "Los fracasos del chantaje: régimen de prohibición de drogas y narcotráfico”, en Mónica Serrano y Arturo Alvarado (eds.), Seguridad Nacional y Seguridad Internacional: Los grandes problemas de México, vol. xv, México, D.F., El Colegio de México, 2010, pp. 62-104. Schievenini, José Domingo, "La criminalización del consumo de marihuana en México (1912-1961)", tesis de doctorado, México, UNAM, 2018.

${ }^{12}$ Campos, art. cit. 
ción y el caos comunicativo. Aunque en lo general el presente artículo coincide con el cuidadoso análisis histórico de Campos, no nos parece que, incluso una defensa impecable de la diplomacia mexicana hubiera sido suficiente para evitar la suspensión del Reglamento; esto es, no estamos necesariamente ante un fracaso diplomático, como sugiere el título de su artículo. Por lo demás, nuestro artículo pretende ofrecer una visión más amplia de le negociación pues, mientras Campos limita su análisis prácticamente sólo al primer semestre de 1940, nosotros examinamos también las conversaciones formales e informales que ocurrieron entre representantes de la Oficina de Aduanas y el Dsp desde abril de 1938.

Un segundo texto que ha analizado con cuidado el proceso de negociación es la tesis de licenciatura de Mariana Flores Guevara, quizás el texto que mejor pone en contexto este importante momento de la diplomacia mexicana. Entre sus principales virtudes, la tesis presenta un puntual análisis de la sesión del Comité Consultivo sobre el Tráfico de Opio y otras Drogas Peligrosas (OAC) celebrada en Ginebra durante el verano de 1939, así como de los principales acontecimientos ocurridos durante el primer semestre de $1940 .{ }^{13}$ Flores Guevara, sin embargo, excluye de su análisis prácticamente todo lo acontecido en el año 1938, y sólo reconstruye el proceso de negociación con base en la mirada mexicana, recogida en los documentos del Archivo Diplomático Genaro Estrada; todo lo relativo a la perspectiva estadounidense lo obtiene de fuentes secundarias, en las que destaca el artículo de William Walker. ${ }^{14}$

Este último texto, publicado en la década de los años setenta, fue el primero en acercarse, si bien de forma breve, a la disputa diplomática entre México y Estados Unidos en tor-

${ }^{13}$ Flores Guevara, "La alternativa mexicana al marco internacional de prohibición de drogas durante el Cardenismo".

${ }^{14}$ William Walker III, "Control across the Border: The United States, Mexico, and Narcotics Policy, 1936-1940”, Pacific Historical Review 47, núm. 1 (1978), pp. 91-106. 
no al Reglamento de Toxicomanías. Walker tuvo acceso exclusivo a los archivos del Departamento de Estado y con base en ellos reconstruyó la negociación diplomática. El texto, sin embargo, no tomó en cuenta ninguna fuente mexicana y sus conclusiones resultan, necesariamente, parciales.

Además de presentar el análisis de documentos hasta ahora no analizados, este artículo ofrece un diálogo crítico con la evidencia e ideas planteadas en los tres textos anteriores. Para ello, reconstruye la relación transgubernamental alrededor del tema a partir de fuentes primarias recuperadas en archivos en México y Estados Unidos, incluyendo el Archivo General de la Nación, el Archivo Histórico Diplomático Genaro Estrada, los archivos personales de Harry Anslinger depositados en la Librería de la Universidad de Pennsylvania, y los Archivos Nacionales de EE.UU, localizados en Maryland. Al revisar todo el material conocido sobre este proceso, el texto es, hasta donde sabemos, el primero en reconstruir los pormenores de la relación transgubernamental con respecto al tema del Reglamento de Toxicomanías de 1940 en su conjunto.

Al presentar un examen pormenorizado de un episodio fundamental en la política de drogas en México, el artículo contribuye al estudio de la relación México-Ee.uU. en materia de narcóticos, en particular, al examen de la toma de decisiones de EE.UU. con respecto a la política interna mexicana. Asimismo, busca contribuir al estudio histórico de la política de drogas en México, un campo que en la última década ha dado pie a un importante corpus de trabajos. ${ }^{15}$

Lo que resta del texto se divide en tres partes. En primer lugar, se revisa la reacción estadounidense ante el plan de Salazar, desde el momento en que la Oficina de Aduanas se entera por cuenta del propio Salazar del proyecto de reforma en abril de 1938, hasta que éste es trasladado a la Oficina de la Presidencia de la República para ser firmado por Lázaro

15 Véase, por ejemplo, el número especial dedicado a la historia de las drogas y sus políticas publicado en The Social History of Alcohol and Drugs: An Interdisciplinary Journal, vol. 43, ejemplar 1, 2000. 
Cárdenas el 5 de enero de 1940. En segundo lugar, se revisa lo acontecido en el primer semestre de 1940, esto es, la reacción estadounidense frente a la promulgación del Reglamento. Termina en julio de 1940, cuando se suspende el Reglamento por tiempo indefinido. Por último, la conclusión ofrece una síntesis de los principales hallazgos del artículo y presenta nuevas vetas de análisis para futuras investigaciones.

\section{La ReAcción ESTAdounidense ANTE EL PLAN de SAlazar}

La Oficina de Aduanas de EE.uU. se enteró de las intenciones de Salazar de crear un monopolio estatal de distribución de narcóticos en abril de 1938. Específicamente, gracias a una entrevista entre su agente Harold S. Creighton y el mismo Salazar.

Sin un funcionario permanente en México, la Oficina de Aduanas, una burocracia anclada en el Departamento del Tesoro, era la instancia encargada de resolver todos los asuntos relacionados con el tema de drogas con México. Creighton, un viejo agente aduanal con residencia en Texas, viajaba varias veces al año a la Ciudad de México para resolver asuntos relacionados con el problema del narcotráfico. Salazar era su interlocutor natural y aquella reunión de abril de 1938 fue la primera en que los dos funcionarios se encontraron para revisar la agenda común.

Aunque en aquella entrevista no se habló de una posible reforma a ningún reglamento, Salazar sí le manifestó a Creighton su opinión con respecto al tema del tráfico de drogas resumida en una sola frase: sólo puede detenerse el poder corruptor de la droga a partir de la creación de un monopolio estatal. ${ }^{16}$ Tan impresionado quedó Creighton de la voluntad de Salazar, que en su reporte sobre aquella reunión sugirió buscar la forma de reponer al antecesor de Salazar, Luis G.

${ }^{16}$ James Stewart a Secretary of State, "Narcotics situation in Mexico", 7 de abril de 1938, RG 170, Caja 22, fólder 4, NARA. 
Franco, como jefe de la DTHM, burócrata con quien los estadounidenses habían trabajado de manera coordinada en el pasado. En opinión de Creighton, Salazar era un "médico muy prestigiado, pero no fue enseñado a ser policía”. ${ }^{17}$

¿Por qué causaba ruido la posición de Salazar al funcionario de Aduanas? Proponemos dos razones. Por un lado, la creación de un monopolio estatal de distribución de narcóticos contravenía, desde su perspectiva, los acuerdos internacionales impulsados por EE.UU. desde 1909 y que, ya desde entonces, apuntalaban un enfoque prohibitivo. Asimismo, la posición de Salazar vulneraba la propia legislación mexicana. ${ }^{18}$

Por otro lado, incluso aceptando que había "buenas razones por las cuales un Estado pudiera considerar crear un monopolio de distribución de drogas", en el contexto mexicano de "debilidad estatal e incapacidad para imponer la ley", una propuesta como la de Salazar, según el diagnóstico de Creighton, incentivaría a los traficantes de drogas a dejar de viajar a Europa y Asia para conseguir sus cargamentos y, en su lugar, hacerse de la mercancía por módicos precios directamente en México. ${ }^{19}$ Esto es, se asociaba un (inverosímil) vínculo entre el plan de Salazar y el potencial aumento de la oferta de droga en EE.uU.

A principios de mayo de 1938, el subsecretario de la Oficina de Aduanas, Thos J. Gorman, comunicó al agente Creigthon

${ }^{17}$ Loc. cit.

18 México ingresó formalmente al régimen de prohibición en 1912 al firmar (aunque no ratificar) la Convención de la Haya de ese año. En 1916, se prohibió, a nivel federal, la importación y exportación de opio. En 1920 se promulgaron las disposiciones "sobre el comercio de productos que pueden ser utilizados para fomentar vicios que degeneren la raza y sobre el cultivo de plantas que pueden ser empleadas con el mismo fin". Estas disposiciones proscribieron el cultivo y comercio de marihuana y opio en todo el país. Finalmente, en 1923, el presidente Obregón extendió la proscripción a la exportación de otras sustancias, incluyendo la cocaína y derivados, heroína y morfina.

${ }^{19}$ Las citas anteriores provienen de H.S. Creighton a Commissioner of Customs, 15 de junio de 1938, RG 170, Caja 22, fólder 4, NARA. 
que ya había contactado con el Departamento de Estado para buscar la reinstalación de Franco. La presión se haría directamente al general Lázaro Cárdenas por medio del Consulado de México y del exjefe del DsP, José Siurob. ${ }^{20}$ La restitución de Franco se presentaba como una salida óptima, no sólo por la cooperación que se había mantenido con él durante su periodo como predecesor de Salazar, sino porque Franco se había convertido en un transmisor de información de la Oficina de Aduanas; fue él quien, confidencialmente -¿quizás buscando su eventual reinstalación?- entregó a Creighton ese mismo mayo un borrador de las reformas que pensaba llevar a cabo Salazar. En justificación no solicitada, argumentó que lo hacía en el afán de mostrar respeto a los tratados internacionales firmados por México. ${ }^{21}$

Sería exagerado afirmar que, en esta primera etapa, el tema fuese prioritario para la embajada y la diplomacia estadounidense. La agenda mexicana estaba todavía paralizada por el decreto de expropiación petrolera del 18 de marzo de 1938. Aun así, es de resaltar la atención que desde el inicio pusieron los estadounidenses en este tema en los meses siguientes. A continuación, un breve recuento.

Además de buscar la reinstalación de Franco - proyecto hasta ese momento infructuoso- Creighton propuso a Harry J. Anslinger, el comisionado de narcóticos de EE.UU., y un personaje clave en esta historia, invitar a Salazar a Washington para que ambos mantuvieran una conversación formal. Según el cálculo del agente aduanal, una invitación a la capital estadounidense permitiría socializar al médico mexicano, hacerle ver la relevancia de observar las leyes antinarcóticos y quizás así convencerlo de que, si bien sus ideas eran correctas en lo teórico, estaban fundamentalmente equivocadas. ${ }^{22}$

${ }^{20}$ Thos J. Gorman a H.S. Creighton, 17 de mayo de 1938, RG 170, Caja 22, fólder 4, NARA.

${ }^{21}$ Luis G. Franco a H. S. Creighton, "[confidential]", 24 de mayo de 1938, RG 170, Caja 22, fólder 4, NARA.

${ }^{22}$ Creighton a Commissioner of Customs, 15 de junio de 1938. 
Aunque todo apunta a que Salazar sí fue a Estados Unidos en los meses siguientes, no tenemos evidencia de que ambos funcionarios se hayan encontrado. Sabemos, sin embargo, que Anslinger le hizo llegar una serie de documentos que explicaban cómo su oficina en EE.Uu. abordaba el problema de la droga y cómo, desde esa perspectiva, no había espacio para experimentos como el que promovía Salazar. ${ }^{23}$

De forma paralela, funcionarios de la Oficina de Aduanas comenzaron a filtrar información a diferentes medios de comunicación estadounidenses sobre un supuesto incremento de campos sembrados de amapola en México. Así, por ejemplo, en declaraciones al Washington News, Creighton llamó la atención a Salazar por la poca atención que el DsP prestaba al tema. El contraste era evidente, pues sólo hacía unos meses -cuando aún no se tenía constancia del interés de Salazar por reformar el Reglamento de Toxicomanías- de la voz y pluma del agente de la Oficina de Aduanas únicamente provenían loas y buenas palabras al trabajo del DsP. ${ }^{24}$ Claramente la situación había cambiado.

Los últimos días de julio, un periódico de San Antonio, el San Antonio Light, reportó el plan de Salazar de establecer un monopolio estatal de narcóticos. ${ }^{25}$ En agosto, otro periódico, The Dallas Dispatch, retomó la información y publicó un artículo titulado Dangerous Proposal. ¿Quién filtraba la información si el plan continuaba cocinándose solamente en el DSP? Desde la propia Oficina de Aduanas, se especulaba que la filtración podría haber venido de su oficina regional de San Antonio, algo que su supervisor negó. ${ }^{26}$

${ }^{23}$ Harry J. Anslinger, "Memorandum for Mr. Bulkley", 7 de diciembre de 1938, RG 170, Caja 22, fólder 4, NARA.

24 Harry J. Anslinger a Leónides Andreu Almazán, 20 de abril de 1938, RG 170, Caja 22, fólder 4, NARA.

25 "U.S. Protests mexican plan to sell dope", The San Antonio Light, 31 de julio de 1938, RG 170, Caja 22, fólder 3, NARA.

${ }^{26}$ H.S. Creighton a Commissioner of Customs, 31 de agosto de 1938, RG 170, Caja 22, fólder 4, NARA. 
Durante el otoño de 1938 no dejó de crecer la preocupación de las autoridades estadounidenses; sin embargo, entre el Departamento de Estado, el comisionado Anslinger, y la Oficina de Aduanas no se alcanzaba ningún consenso en cómo abordar la situación. ¿Habría que hablar con Salazar de forma confidencial? ¿Tendría sentido, como apuntó Creighton, invitarlo y halagarlo de nuevo en EE.UU.? $¿$ ¿Se debía privilegiar el mantener la relación estable ante la perspectiva de una situación de guerra contra Alemania? ¿Cuál de las tres burocracias debía tomar el mando de la estrategia?

Todavía sin una hoja de ruta clara, el 6 de septiembre, el cónsul general James Stewart y el agente aduanal Alvin Scharff -subordinado de Creighton y quien en abril había acompañado a militares y a agentes sanitarios en las campañas de erradicación en el sur de Sonora- se reunieron con el jefe del DSP, Leónides Almazán, con el propósito de tratar el tema directamente con él; éste los envió directamente a hablar con Salazar. Casi a modo de broma, les aconsejó que tuvieran cuidado con lo que el doctor les ofreciera al llegar a su oficina, pues "Salazar solía recibir a sus visitar con cigarros de marihuana". ${ }^{27}$ Broma o no, el comentario no debió tranquilizar demasiado a los estadounidenses.

Un día después, el 7 de septiembre, Scharff se reunió en privado con Salazar y pudo comprobar la seriedad y el ímpetu que el médico imprimía a su propuesta. ${ }^{28} \mathrm{~A}$ sabiendas de que en verano ya se había filtrado un primer documento a la prensa, Salazar le dio a Scharff un borrador de su proyecto, así como un bosquejo de la receta médica que se tenía contemplada para la entrega de narcóticos por los médicos registrados ante el DSP. Aunque reconocía su obvia inteligencia y preparación, al redactar el memorándum de la conversa-

${ }^{27}$ H. S. Creighton a Commissioner of Customs, 15 de septiembre de 1938, RG 170, Caja 22, fólder 4, NARA.

${ }^{28}$ Creighton a Commissioner of Customs. 
ción, Scharff calificó las posiciones del doctor mexicano como "completamente anticatólicas". 29

Ante una situación francamente complicada, los estadounidenses habían encontrado poderosos e inesperados aliados en México. En agosto, un miembro anónimo del Comité Nacional Auxiliar del Dsp filtró a Creighton que Salazar, en reunión plenaria, había ofrecido cigarrillos de marihuana a los presentes. Más aún, él mismo habría fumado varios. ${ }^{30}$ Asimismo, surgieron señalamientos que lo acusaban de haber distribuido cigarrillos "de cama en cama" a sus pacientes en el hospital de la Castañeda. ${ }^{31}$ Conforme pasaban los meses, también los diarios Excélsior y El Nacional comenzaron a hacer eco de las voces de funcionarios del DsP que renegaban de las tesis de Salazar y que no ahorraban adjetivos para referir su aparente locura. ${ }^{32}$ Mientras tanto, el presidente de la Asociación Mexicana de Neurología y Psiquiatría criticó públicamente la posición de Salazar con respecto a la marihuana, la cual, según el médico duranguense, hacía mucho menos daño de lo que se decía. ${ }^{33}$ Algún abogado sugirió que, de respetarse el Código Sanitario vigente, Salazar debía ir preso por

${ }^{29}$ Alvin F. Scharff, "Memorandum for Consul General James B. Stewart concerning Conversation had by the undersigned with Dr. Leopoldo Salazar Viniegra", 7 de septiembre de 1938, RG 170, Caja 22, fólder 4, NARA.

30 Supervising Customs Agent H.S. Creighton a Commissioner of Customs, 19 de agosto de 1938, RG 170, Caja 22, fólder 4, NARA.

${ }^{31}$ Embajada de Estados Unidos en México, "Serious charges against Salazar Viniegra", 1 de noviembre de 1938, RG 170, Caja 22, fólder 4, NARA. Véase James Stewart a Secretary of State, "Accusations against Dr. Salazar Viniegra [confidential]", el 15 de noviembre de 1938, RG 170, Caja 22, fólder 4, NARA.

32 "Laws and Marihuana: The discussion as to whether or not it is a toxic herb has presented a very complicated situation (versión traducida)", El Nacional, 25 de octubre de 1938, RG 170, Caja 22, fólder 4, NARA; "Penalties for smoking marihuana. The Department of Public Health is not in agreement with the thesis of Dr. Salazar Viniegra (traducción)", Excélsior, 27 de octubre de 1938, RG 170, Caja 22, fólder 4, NARA.

${ }^{33}$ Manuel Guevara Oropeza, "Marihuana is dangerous: opinion of an eminent neurologist", El Universal, 24 de octubre de 1938, RG 170, Caja 22, fólder 4, NARA. 
hacer apología a la marihuana en sus conferencias y escritos médicos. ${ }^{34}$

Respecto a esto, es de resaltar que en los periódicos mexicanos lo que llamaba la atención no era la propuesta de crear un monopolio estatal de distribución de morfina, sino las posiciones sui géneris que mantenía Salazar sobre la marihuana. Cabe proponer la hipótesis de que las dos agendas se superpusieron y resultaron indistinguibles para el gran público -lo cual sigue siendo cierto hoy: muchos artículos periodísticos recientes han sugerido que Salazar legalizó la marihuana en México en 1940-. ${ }^{35}$ Esa confusión no sólo lastimó la legitimidad de Salazar en tanto promotor de la propuesta, sino que redujo considerablemente sus posibilidades de éxito.

Harry Anslinger, quien hasta ese momento había tenido una posición secundaria, supo aprovechar a sus aliados mexicanos, sobre todo a los periódicos y médicos opositores a Salazar. Al igual que lo hizo en su país, donde en 1937 inició una campaña publicitaria para promover la ley federal que prohibió la marihuana a nivel federal, en México utilizó todos los medios posibles para avanzar sus ideas. Así, por ejemplo, la revista Jueves de Excélsior publicó un artículo de Anslinger titulado "Mariguana, asesino de la juventud" y que, según los editores de la revista, "servía para contradecir a Salazar en el sentido de que la mariguana era inofensiva”. ${ }^{36}$ En los meses posteriores buscó El Universal la publicación de las opiniones de al menos dos médicos extranjeros que rebatieran las ideas de Salazar. ${ }^{37}$ Tan importante como ganar

34 "Laws and Marihuana", ibid.

35 Véase, por ejemplo: Katia D’Artigues, "El presidente que legalizó las drogas", El Universal, 10 de febrero de 2017.

36 No me fue posible encontrar el artículo en su versión original. Sobre esta afirmación sigo lo apuntado por Pérez Montfort, op. cit. p. 288.

${ }^{37}$ Nos referimos al tunecino J. Bouquet y al estadounidense James C. Munch. Véase Harry J. Anslinger a J. Bouquet, 30 de diciembre de 1938, RG 170, Caja 22, fólder 2, NARA.; Harry J. Anslinger a James C. Munch, 25 de abril de 1939, RG 170, Caja 22, fólder 4, NARA. 
el debate diplomático era, para Anslinger, ganar el espacio público.

Quizás para reducir la confrontación, en noviembre de 1938 Salazar escribió al agente Scharff pidiéndole información sobre lo que "gente inteligente" de EE.uU. pensaba sobre el tema de la marihuana. Si era posible, pedía que le mandasen toda la información que tuviera el gobierno de EE.UU. sobre la hierba para considerarla en sus estudios. Scharff interpretó esa petición como una reacción de Salazar frente a la prensa negativa que había aparecido contra él en las últimas semanas. ${ }^{38}$

Anslinger envío los materiales solicitados, pero la expectativa de Scharff resultó no estar fundamentada. Salazar siguió adelante.

El agente Creighton había llegado a la conclusión de que nada podía lograrse mientras Salazar continuara empleado. A pesar de eso, cada vez que viajaba a México desde su residencia en San Antonio, éste llamaba a Salazar para tocar base e intentar averiguar sobre los avances del médico mexicano. En enero de 1939 llegó a juntarse hasta tres días consecutivos con él. Así fue como descubrió que sus oficinas habían cambiado a la calle Sevilla número 33 -sitio donde se establecería el dispensario un año después-, que a su mando sólo había tres policías y que Salazar no parecía muy interesado en destinarlos para tareas antinarcóticos. ${ }^{39}$

El 2 de marzo de 1939, en un acto acaso desafortunado, Salazar envió por correo a Anslinger dos ejemplares de su estudio sobre la marihuana, así como dos folletos de "Doña Juanita”, panfleto que burlonamente intentaba desmitificar los daños de la hierba. ${ }^{40}$ Si no lo hizo para provocar al comisionado estadounidense, difícilmente se entiende tal candidez política.

38 Alvin F. Scharff a Commissioner of Customs, 22 de noviembre de 1938, RG 170, Caja 22, fólder 4, NARA.

${ }^{39}$ H. S. Creighton a Commissioner of Customs, 21 de febrero de 1939, RG 170, Caja 22, fólder 4, NARA.

${ }^{40}$ Leopoldo Salazar Viniegra a Harry J. Anslinger, 2 de marzo de 1939, RG 170, Caja 22, fólder 4, NARA. 


\section{La 24. ${ }^{a}$ sesión del Comité Consultivo sobre el Tráfico de Opio y otras Drogas Peligrosas}

En un clima de franca animadversión y confrontaciones indirectas llegó la primavera de 1939 y, con ello, la 24. ${ }^{a}$ sesión del Comité Consultivo sobre el Tráfico de Opio y otras Drogas Peligrosas (OAC), la instancia de la Liga de las Naciones dedicada al tema antinarcóticos a escala global. La reunión, a la que asistieron Anslinger y Salazar como parte de sus respectivas delegaciones, se celebró en Ginebra entre el 15 de mayo y el 12 de junio de $1939 .{ }^{41}$ Se trató de la última cumbre antes de que por la Segunda Guerra Mundial se suspendieran las operaciones de la Liga de las Naciones.

Apenas llegar, de manera informal, el 27 de mayo, Stuart Fuller -encargado del Departamento de Estado para asuntos antinarcóticos- se reunió con Salazar y el delegado mexicano Jorge Deassle en un hotel ginebrino. En ese encuentro, Fuller intentó persuadir al doctor mexicano de lo equivocado de su plan y del mal recibimiento que éste tendría en la conferencia; el objetivo de Fuller era evitar a toda costa que la delegación mexicana presentase su plan en la sesión del comité. ${ }^{42}$ La sola posibilidad de que sus ideas tuvieran un foro internacional de ese calibre era un inconveniente suficiente. En el reporte sobre aquella reunión, Fuller calificó a Salazar de "novato", "inexperto" y de carácter "inestable"; además, quizás pensando en Deassle, recalcó que los colegas mexicanos de Salazar no parecían muy impresionados por sus tesis pues, en otros contextos, afirmaban que éstas no representaban las del resto de la delegación. ${ }^{43}$ Por último, en el reporte se aconsejaba que si el plan de Salazar se ponía

${ }^{41}$ Los delegados permanentes del Comité por parte de México fueron Manuel Tello y Jorge Deassle; Salazar asistió en condición de delegado sustituto.

42 Rúbrica ilegible, "Memorandum of conversation with Dr. Salazar Viniegra in the presence of Mr. S.J. Fuller and a representative of the Mexican Delegation”, 27 de mayo de 1939, RG 170, Caja 22, fólder 4, NARA.

${ }^{43}$ Loc. cit. 
finalmente en funcionamiento, la policía mexicana "debería comprarle de forma subrepticia la droga a todos los adictos que obtuvieron su morfina de las clínicas para así mostrar lo poco funcional del plan de Salazar". ${ }^{44}$ Años después, Salazar recordaría esa misma reunión y diría que en ella se acordó que "nuestro sistema no se pondría en vigor hasta que, ulteriormente, en conferencias que tendrían lugar en Estados Unidos o en México, se allanaran los inconvenientes”, cosa que no sucedió. ${ }^{45}$

Las fracturas internas en la delegación mexicana, ya avizoradas en el reporte citado, quizás expliquen el que Salazar no leyese la posición mexicana en la sesión formal del OAC tal como estaba planeado. El hecho es que en algún momento entre finales de mayo y principios de junio, Salazar, que había viajado a Suiza como delegado sustituto, justamente para explicar los pormenores de la propuesta mexicana, desapareció misteriosamente del mapa, así como de cualquier documento.

Fue Manuel Tello, delegado mexicano permanente en Ginebra, el encargado de hacer lectura de un texto preparado expresamente por el doctor duranguense para la ocasión. Tanto el discurso principal, que tuvo lugar el 16 de mayo, como los argumentos de defensa presentados en tres sesiones posteriores parecen haber sido respetados en lo general por el diplomático. Tello anunció formalmente que México preparaba nuevas leyes para el tratamiento de toxicómanos y explicó brevemente el procedimiento: éstos podrían adquirir de forma lícita la cantidad de drogas recetadas por los médicos responsables. Destacó que más allá de ser una solución al problema de adicción, lo que se buscaba era mantener a los consumidores alejados de las cadenas de dependencia controladas por los traficantes. En ese sentido, dijo el diplomático mexi-

${ }^{44}$ Loc. cit.

45 Salazar Viniegra, “Opio y política: Historia de una humillación”, art. cit. 
cano, se estaría dando un paso al frente contra el tráfico de drogas, y no a su favor. ${ }^{46}$

Como era de esperarse, la posición mexicana dio pie a la respuesta de Anslinger. El comisionado estadounidense señaló que, de aprobarse los nuevos lineamientos, México no sólo estaría atentando contra el espíritu de la Convención de Ginebra de 1925, sino dando pie a una política equivocada. Como yuxtaposición a la propuesta mexicana, Anslinger expuso como modelos las granjas e instituciones psiquiátricas que atendían a los toxicómanos estadounidenses. ${ }^{47}$ Sus discursos encontraron eco en la delegación canadiense cuyo representante descalificó con igual o mayor vehemencia la posición mexicana. Arrinconado, sin apoyo explícito de otras delegaciones, y sin la presencia de Salazar, Tello acordó llevar las preocupaciones de vuelta a México y volver a discutir el tema el año siguiente.

No es claro cuándo vuelve Salazar a México ni cómo explica su ausencia en las discusiones de Ginebra. Lo cierto es que en agosto de 1939, Leónides Andreu Almazán y Leopoldo Salazar renunciaron a sus cargos. Fueron reemplazados, respectivamente, por José Siurob y Heberto Alcázar. No parece que tal reorganización tuviese que ver con la propuesta de Salazar ni con su misteriosa desaparición en Ginebra; la explicación más probable está relacionada con el anuncio de la candidatura opositora a la presidencia del hermano de Leónides, Juan Andreu Almazán. ${ }^{48}$ El propio Salazar, en carta a Anslinger, no culpa a la presión estadounidense de su destitución sino al hecho de que "en México los asuntos técnicos

46 Manuel Tello, "Advisory Committee on Traffic in Opium and Other Dangerous Drugs, $24^{\circ}$ Session, Minutes of the Twentieth Meeting", 3 de junio de 1939, NARA.

${ }^{47}$ Para una revisión de los argumentos de Anslinger durante la sesión, y en general para un análisis de lo sucedido en Ginebra, consúltese: Flores Guevara, "La alternativa mexicana al marco internacional de prohibición de drogas durante el Cardenismo", cap. v, op. cit.

${ }^{48}$ Juan Andreu Almazán hizo pública su candidatura el 28 de julio de 1939. El 4 de agosto fue destituido Leónides como jefe del DSP. 
están atados a la política". ${ }^{49}$ Del mismo modo, en un artículo publicado en Excélsior años después, acusó a la "eterna y funesta política electoral" de la separación del cargo. ${ }^{50}$

Todos los actores estadounidenses involucrados supusieron que la vuelta de José Siurob a la dirección del Dsp era el punto final a la propuesta de tratamiento ambulatorio de adicciones. Las primeras acciones de Siurob parecían confirmar esas sensaciones y avisaban cierta tranquilidad: Siurob cambió la retórica de Salazar -"los adictos son inadaptados", 51 afirmó en una conferencia- y llegó a escribir al propio Anslinger en noviembre de 1939 pidiéndole información sobre "los métodos empleados por los Estados Unidos en la educación de sus agentes antinarcóticos”. Más aún, lo invitó a visitar México con el fin de discutir posibles colaboraciones en el área de entrenamiento policial. ${ }^{52}$

Ante el supuesto de que Siurob cambiaría el rumbo del DSP en lo que respecta a su política antidrogas, Creighton sugirió el envío a Siurob de todos los panfletos posteriores a 1924 sobre el consumo de narcóticos en EE.UU. y los factores que lo inducen, una publicación sobre el funcionamiento de centros de rehabilitación, un reporte de un médico cercano al FBN sobre su experiencia en Egipto, así como otros documentos que constituían el corpus básico de conocimiento sobre drogas y adicción con el que la Oficina de Aduanas y el FBN argumentaban su posición contra los tratamientos de políticas de reducción de daños. ${ }^{53}$ Todo parecía volver a la normalidad.

49 Véase Leopoldo Salazar Viniegra a Harry J. Anslinger, 29 de abril de 1940, RG 170, Caja 22, fólder 4, NARA.

50 Salazar Viniegra, "Opio y política: historia de una humillación”, art. cit.

51 José Siurob, "The Struggle against Toxicomanías", International Conference of Executives of the Law of the Pacific Coast, Ciudad de México, Bellas Artes, 1939.

${ }^{52}$ José Siurob a Harry J. Anslinger, 30 de noviembre de 1939, RG 170, Caja 22, fólder 2, NARA.

53 Supervising Customs Agent H.S. Creighton a Commissioner of Customs, 17 de enero de 1940, RG 170, Caja 22, fólder 2, NARA. 


\section{LA REACCIÓN ESTADOUNIDENSE FRENTE A LA PROMULGACIÓN DEL REGLAMENTO}

Es verdad que la Segunda Guerra Mundial había estallado. También es cierto que las prioridades de la embajada y del consulado habían cambiado y que quizás asegurarse de la lealtad mexicana ante la crisis europea era una prioridad. Y sin embargo, ¿cómo explicar que los estadounidenses no se percataran de que el plan de tratamiento ambulatorio de adicciones continuaba procesándose en el DSP? El hecho es que los tomó por sorpresa pues nada de la correspondencia sostenida entre octubre y diciembre de 1939 hacía referencia al tema.

A finales de 1939 la propuesta de reforma al Reglamento de 1931 estaba terminada y llegó a la mesa del general Lázaro Cárdenas el 5 de enero de 1940. El 17 de febrero fue publicada la reforma y tres semanas después, el 9 de marzo, se había abierto el dispensario de la calle Sevilla.

La Embajada de EE.uU. se enteró de lo avanzado del plan a finales de diciembre de 1939 cuando, gracias a la filtración de Alberto P. León, un alto funcionario del DsP y miembro del Consejo de Salubridad General, recibió una copia de la propuesta del Reglamento. ${ }^{54}$ Las vacaciones de fin de año retrasaron la respuesta diplomática.

El 9 de febrero de 1940 el ministro plenipotenciario de la Embajada de México en Washington, Luis Quintanilla, se entrevistó en esa ciudad con el jefe de la División de las Repúblicas Americanas del Departamento de Estado, Laurence Duggan. En ese primer encuentro, Duggan le comunicó al diplomático mexicano que, a ojos del Departamento de Estado, la reforma mexicana "difiere completamente de las que rigen en todos los países del mundo que son parte de las Con-

${ }^{54}$ Josephus Daniels a The Secretary of State, "Forwarding Mexican Regulations regarding Toxicamania (Drug Addiction)", 30 de diciembre de 1939, RG 170, Caja 22, fólder 2, NARA. 
venciones Internacionales sobre Narcóticos". ${ }^{55}$ Por tanto, continuó Duggan, en caso de que México continuara con la implementación de la reforma, el gobierno de EE.UU. apelaría a la Ley de Importación y Exportación de Drogas Narcóticas de ese país -una ley originalmente escrita en 1922- que prohibía a las personas o empresas sujetas a la jurisdicción estadounidense exportar drogas narcóticas (principalmente morfina y codeína) a países que, a juicio del comisionado de narcóticos, no mantuvieran un sistema de control conforme a la Convención Internacional del Opio de 1912. En la práctica, amenazaba Duggan, esto permitiría a Anslinger negar licencias de envíos legales de narcóticos a México en tanto que el país incumplía sus obligaciones internacionales y no garantizaba el uso médico de las importaciones. Duggan terminaba diciendo que: "Por encomiable que parezca la intención del proyecto de ley mexicana de proporcionar narcóticos a los adictos con el único fin de satisfacer su necesidad, el comisionado de narcóticos no podría considerar este acto más que como una distribución de estupefacientes para satisfacción del vicio $[\ldots] " .56$

El embajador mexicano en Washington, Francisco Castillo Nájera, envió a México un reporte de aquel encuentro con una nota urgente en la que llamaba "la atención de nuestras autoridades sanitarias, y demás personas interesadas, sobre la seriedad de las objeciones del gobierno americano". ${ }^{57}$ Isaac Campos calificó como un "retraso catastrófico de comunicación para la cual la burocracia de la SRE no puede tener excusas", 58 dado que el memorándum de la reunión entre Duggan y Quintanilla no llegó a la mesa del jefe del DSP sino

55 F. Castillo Nájera a Secretario de Relaciones Exteriores, "Convención Internacional sobre Estupefacientes. Restricciones a la importación desde Estados Unidos", 9 de febrero de 1940, Topografía III -2398-6, Archivo Histórico Genaro Estrada.

56 Loc. cit.

${ }^{57}$ Loc. cit.

${ }^{58}$ Campos, art. cit., p. 5. 
hasta el 9 de marzo, esto es, un mes después de celebrada la reunión.

El retraso de comunicaciones tuvo la consecuencia desafortunada de que el DSP continuó con los planes originales sin tomar en cuenta las señales que desde Washington se enviaban a los responsables en México de tomar decisiones. ${ }^{59}$ Entretanto, a principios de marzo, Creighton viajó a la Ciudad de México para entrevistarse con Siurob y Alcázar. En otro inexplicable retraso, al agente estadounidense lo hicieron esperar varios días antes de concertar una cita. Conocemos de la frustración de Creigthon por sus cartas a sus superiores. Sabemos también que a Heberto Alcázar le habría hecho una oferta extra para convencerlo de derogar el reglamento ("Made him another offer-which I will not put on paper and probably not ever tell you about [...]). La frustración con los mexicanos claramente despertaba los peores instintos del agente estadounidense ("I wanted to commit a few murders -but I have now recovered"). ${ }^{60}$

Mientras todo eso sucedía, al son de la Banda de Música del Estado Mayor, la mañana del 9 de marzo abrió el dispensario. Todavía Siurob no era consciente de la amenaza que se cernía sobre México, sin embargo, esa misma tarde se le hizo llegar al jefe del DSP, con un mes de retraso, el memorándum del 9 de febrero firmado por el embajador Castillo Nájera. ${ }^{61}$

Lo primero que hizo Siurob fue llamar a sesionar el 12 de marzo, de manera urgente, al Consejo de Salubridad General. Sabemos de la seriedad con que los 23 miembros de

${ }^{59}$ Loc. cit.

${ }^{60}$ Ambas citas entrecomilladas pertenecen a: H. S. Creighton a E. J. Shamhart, "[confidential]", 5 de marzo de 1940, RG 170, Caja 22, fólder 2, NARA.

61 José Siurob a Secretario de Relaciones Exteriores, "Sobre notas enviadas por el Gobierno americano en relación al nuevo Reglamento de Toxicomanías expedido por nuestro Gobierno", 14 de marzo de 1940, Topografía III -2398-6, Archivo Histórico Genaro Estrada. 
ese ente trataron el tema gracias a las actas de sesión. ${ }^{62}$ En aquella reunión, por ser "un asunto urgente de tratar", se leyeron las noticias que habían llegado desde Washington. Hubo voces discordantes en cuanto a la obligación o no de llevar el caso a la Liga de las Naciones, pero en lo general hubo consenso en que el Reglamento había funcionado bien al punto de haber "disminuido el tráfico ilícito de enervantes notablemente", una afirmación aventurada a tres días de haberse puesto en marcha el dispensario. ${ }^{63}$ Se acordó crear una comisión que estudiase el tema a fondo.

Dos días después, el 14 de marzo, llegó a México la comunicación de que Anslinger, en efecto, había decidido imponer el embargo medicinal a México. Ese mismo día, ya con la noticia conocida por todos, volvió a sesionar el Consejo de Salubridad General. Las palabras, muy animadas dos días atrás, ya no lo eran tanto. José Siurob culpó veladamente al "anterior titular del Departamento" al señalar que éste “dejó pendiente la expedición del reglamento y [...] no dejó huella alguna al respecto [...]" y opinó que debía "contestarse que se ha anulado la vigencia del reglamento, para ver si así es posible hacer cambiar el criterio del Gobierno americano". ${ }^{64}$ El doctor Alberto P. León, quien en diciembre había filtrado a la embajada una copia del reglamento, sugirió "afrontar la realidad [...y] suspender el reglamento". De lo contrario, "antes de un mes se habrían concluido las existencias de drogas, la sanción impuesta a México se hará sentir y se estaría en el caso de no contar con drogas ni para tratamientos médicos urgentes". ${ }^{65}$ Tras cinco horas de discusión y aunque algunos médicos continuaron sosteniendo que debería mantenerse el reglamento porque tenía un "fin noble

${ }^{62}$ Agradezco a Isaac Campos que me compartiera la copia de todas las actas de sesión del Consejo de Salubridad General del periodo 1938-1940.

63 “Acta de sesión, número 9”, 12 de marzo de 1940, Consejo de Salubridad General, Actas de Sesión, Libros, 2, AHSSA.

64 “Acta de sesión, número 10", 14 de marzo de 1940, 10, Consejo de Salubridad General, Actas de Sesión, Libros, 2, AHSSA.

${ }^{65}$ Loc. cit. 
y revolucionario" y que suspenderlo iría "contra el decoro de México", se autorizó a Siurob "tratar privada y ampliamente el asunto con el personal de la Embajada Americana". ${ }^{66}$

El mismo 14 de marzo Siurob decidió escribir a la sRE que:

[...] con el deseo de llegar a un amistoso y completo acuerdo [...] se pide al gobierno de los Estados Unidos que suprima la prohibición de la exportación de enervantes a nuestro país, dejando nosotros, entre tanto, sin aplicación, la parte del reglamento respectivo que faculta al Departamento para autorizar a los médicos el tratamiento de los toxicómanos con dosis de enervantes superiores a las señaladas en la Farmacopea, dejando así, en suspenso, la aplicación de aquellas medidas que han originado la prohibición de la exportación de enervantes a nuestro país. ${ }^{67}$

Además, pidió que se integrase una comisión mixta que solucionara "definitivamente el problema". ${ }^{68}$

A pesar de que ya había comenzado la estrategia de reducción de daños (diplomáticos), Siurob tardó hasta el 19 de marzo en telegrafiar a Lázaro Cárdenas y solicitarle permiso para negociar formalmente con los estadounidenses; adujo que estos "[han] malinterpretado nuestro decreto sobre toxicomanías". ${ }^{69}$ Cárdenas, de gira por el país y aparentemente poco interesado en el tema, concedió la autorización, misma que fue enviada por telegrama dos días después a la oficina de Siurob. ${ }^{70}$

66 "Acta de sesión, número 10".

${ }^{67}$ Siurob a Secretario de Relaciones Exteriores, "Sobre notas enviadas por el Gobierno americano en relación al nuevo Reglamento de Toxicomanías expedido por nuestro Gobierno", 14 de marzo de 1940.

${ }^{68}$ Loc. cit.

${ }^{69}$ Juan Gallardo Moreno a Lázaro Cárdenas del Río, 19 de marzo de 1940, Fondo Presidentes, Lázaro Cárdenas del Río, Caja 363, expediente 422/3, AGN.

${ }^{70}$ Lázaro Cárdenas del Río a Secretario Particular Agustín Leñero, Presidencia de la República, 21 de marzo de 1940, Fondo Presidentes, Lázaro Cárdenas del Río, Caja 363, expediente 422/3, AGN. 
Siurob continuó con sus esfuerzos de negociar una salida amable y presentó al embajador Daniels un cúmulo de alternativas; entre otras, la creación de una posible comisión bilateral que tratara problemas de drogas. ${ }^{71}$ Además, sugirió que podría eliminarse la práctica de entregar la morfina a los adictos y, en su lugar, inyectárselas directamente en clínicas especializadas, propuesta que, de haberse verbalizado así desde el inicio, difícilmente habría ocasionado el conflicto potencial que comenzaba a emerger. Ahora era, quizá, demasiado tarde.

Daniels, en principio, apoyó una política de negociación y se mostró favorable a que Anslinger suspendiera el embargo. ${ }^{72}$ Sin embargo, desde el Departamento de Estado se desechó la propuesta de la comisión bilateral. La posición de Anslinger, tanto como la del Departamento de Estado, era clara: el fin del embargo pasaba por la inmediata derogación de la reforma firmada por Cárdenas en enero. La presión de Anslinger no sólo comenzaba a ganar la partida al gobierno mexicano, sino que, al hacerlo, vencía también a la propia Embajada de EE.uU. que, para evitar que el problema escalase, intentaba encontrar una salida menos radical.

La guerra jugaba a favor del comisionado: un gran porcentaje de los envíos de narcóticos provenientes de Europa y destinados a otros países de América pasaban necesariamente por el puerto Nueva York. De esta forma quedaba en las manos de Anslinger el abastecimiento de precursores químicos para la incipiente industria farmacéutica en México. Por si fuera poco, la industria farmacéutica en Alemania, Francia y España había entrado también en crisis, lo que hacía a México dependiente de la producción estadounidense.

${ }^{71}$ José Siurob a Josephus Daniels, 23 de marzo de 1940, RG 170, Caja 22, fólder 2, NARA.

72 Harry J. Anslinger a Mrs. Maxon, "Item for weekly report of March 23, 1940”, 21 de marzo de 1940, RG 170, Caja 22, fólder 2, NARA. Resulta interesante que Daniels no hiciera ninguna mención sobre este suceso en su monumental biografía. Véase Josephus Daniels, Shirt-Sleeve Diplomat, Chapel Hill, The University of North Carolina Press, 1947. 
El tiempo era el principal aliado de Anslinger quien, en confesión a un funcionario de alto nivel, se jactó de lo infalible de su plan; según él, las autoridades de salud comenzarían a recular conforme se fueran quedando sin medicamentos para "atender a heridos y enfermos" ${ }^{73}$ Resulta interesante que ninguna instancia de gobierno de EE.UU. anunciara públicamente la decisión o produjera una nota diplomática al respecto; la expectativa era que México renunciara a su política a la brevedad, sin necesidad de llevar la situación al plano público. ${ }^{74}$

Aunque tenía la convicción de que el Reglamento no contravenía las convenciones internacionales firmadas por México, ${ }^{75}$ Siurob era consciente de la situación de crisis. Había todavía suficiente resguardo de medicamentos, pero pronto la noticia del embargo se volvería tema de primera plana en los periódicos mexicanos. Por si fuera poco, el embajador Castillo Nájera se mostraba partidario de poner fin al conflicto pues "la perspectiva no era muy halagadora para el DSP"76 y sugería modificar el reglamento "tomando en cuenta los puntos de vista" de EE.UU. ${ }^{77}$

En mensajes que dan cuenta de su propia contradicción, a principios de abril, Siurob pidió a sus colegas del Consejo de Salubridad General "mantenerse en pie de lucha, con un espíritu animoso y con la conciencia plena de que se está procediendo con un fin noble y de acuerdo con los princi-

${ }^{73}$ Harry J. Anslinger a C.H.L Sharman, "[confidential]”, 28 de marzo de 1940, Caja 2, expediente 21, Harry J. Anslinger Papers.

${ }^{74}$ Esa reflexión está originalmente en: Walker III, "Control across the Border", art. cit., p. 102.

75 Secretaría de Relaciones Exteriores, sRe, "Sugestiones de la Secretaría de Relaciones Exteriores sobre la forma en que podrán resolverse los problemas de carácter internacional que ha planteado la aprobación del reglamento vigente de toxicomanías", 22 de abril de 1940, Topografía III -2398-6, Archivo Histórico Genaro Estrada.

76 F. Castillo Nájera a José Siurob, 30 de marzo de 1940, Topografía III -2398-6, Archivo Histórico Genaro Estrada.

77 SRE, "Sugestiones de la Secretaría de Relaciones Exteriores...", art. cit., p. 3. 
pios dictados por la Revolución". ${ }^{78}$ Menos de dos semanas después, el mismo Siurob había confiado a un funcionario de la SRE que el DSP estaba "dispuesto a retirar" el Reglamento mexicano sobre Toxicomanías. ${ }^{79}$

En ese contexto fue que el Departamento de Estado comunicó a la SRE que aceptarían celebrar reuniones con los mexicanos sobre el Reglamento "siempre que dichas pláticas tengan [tuvieran] puramente carácter informal". ${ }^{80}$

Para ese momento, la disyuntiva mexicana no era tanto sobre si abolir el reglamento, sino cómo hacerlo. Lo más importante de todo: el acto no debía interpretarse como un acto de sumisión. Mucho menos de una administración cuya legitimidad provenía -en gran medida- de la autonomía de su política exterior. Así, en el ánimo de cerrar el capítulo, José Siurob viajó a Washington en mayo, junto con el director del Instituto de Higiene, Dr. José Zozaya. ${ }^{81}$

Nada de lo que aconteció en Washington lo sabríamos si nos conformáramos con la exploración de los documentos del Archivo Diplomático Genaro Estrada que callan sobre este asunto. Afortunadamente conocemos lo discutido en aquellas reuniones gracias a los archivos estadounidenses.

\section{Las reuniones de mayo en Washington}

A la reunión del 4 de mayo de 1940 viajaron, por la parte mexicana, Siurob y el Dr. José Zozaya. En representación de Estados Unidos asistieron altos funcionarios de cuatro buro-

78 "Acta de sesión, número 12", 2 de abril de 1940, Consejo de Salubridad General, Actas de Sesión, Libros, 2, AHSSA.

${ }^{79}$ Jefe de la Sección de Asuntos Políticos Agustín Granja Irigoyen a Secretario de Relaciones Exteriores Eduardo Hay, 16 de abril de 1940, Topografía III -2398-6, Archivo Histórico Genaro Estrada.

${ }^{80}$ F. Castillo Nájera a Secretario de Relaciones Exteriores, 1 de abril de 1940, Topografía III -2398-6, Archivo Histórico Genaro Estrada.

${ }^{81}$ Embajador de los Estados Unidos en México Josephus Daniels a Harry J. Anslinger, 26 de abril de 1940, RG 170, Caja 22, fólder 2, NARA. 
cracias distintas, incluyendo la Oficina de Salubridad, la Oficina de Aduanas, el Departamento de Estado y, naturalmente, el FBN representado por el comisionado Anslinger.

En aquella reunión Siurob habló poco. Apenas le dio tiempo de puntualizar cuatro temas. Primero, que el Reglamento había sido ideado en el periodo en el que estuvo fuera de la jefatura de la DTHм. Segundo, que ciertamente, su aplicación debió esperar a un intervalo de estudio más amplio. Tercero, que, a pesar de todo, los resultados del experimento no habían sido del todo malos y que el dispensario había atendido a más de 700 adictos que, de otro modo, habrían conseguido sus narcóticos "de la mafia". Por último, que el funcionamiento de los dispensarios estaba acotado: los usuarios no podían vender la morfina a otros traficantes pues se les inyectaba directamente en la clínica.

Anslinger refutó las estadísticas y fuentes de información de Siurob, y señaló la incompatibilidad de la reforma con las convenciones internacionales. Asumiendo que Siurob no hablaba inglés, le entregó al Dr. Zozaya "que obviamente era favorable a la posición estadounidense" una serie de documentos que esperaba que éste leyese durante el fin de semana. ${ }^{82}$ Acordaron que el mismo grupo se reuniría el 7 de mayo, el lunes siguiente.

El memorándum de la reunión del segundo encuentro muestra un cambio dramático en la posición de Siurob: ${ }^{83}$ hizo acto de constricción. En voz del Dr. Zozaya "señaló que tras haber leído con cuidado los documentos entregados por Anslinger, reconsideró su posición, se convenció de que EE.UU. tenían razón y que las regulaciones mexicanas estaban completamente equivocadas". ${ }^{84}$ Dijo también que recomendaría

${ }^{82}$ Herbert Gaston, "Conference held on may 4, 1940, 1940 at Washington D.C., for purpose of discussing the narcotic regulations recently enacted in Mexico", 4 de mayo de 1940, RG 170, Caja 22, fólder 2, NARA.

83 Herbert Gaston, "Conference held on same subject on May 7, 1940, in office of Mr. Stuart J. Fuller, Department of State”, 7 de mayo de 1940, RG 170, Caja 22, fólder 2, NARA.

${ }^{84}$ Loc. cit. 
al presidente Cárdenas la inmediata suspensión del reglamento. Eso sí, pidió no avisar a los medios de comunicación de tal decisión ni producir una declaración formal; esto con el fin de no dar a los críticos la oportunidad de ridiculizar a su departamento. Se dio tiempo para exponer el motivo principal de su preocupación: porque en tiempos de elecciones no debe darse la impresión de que EE.uU. "mandan otra vez". ${ }^{55}$

Anslinger, complacido con la posición de Siurob, explicó cómo la ley Harrison había vuelto corruptibles a los médicos que prescribían drogas y cómo las clínicas de atención a adictos habían fracasado en su cometido. Además, ante la tímida idea de Siurob de mantener viva la posibilidad de ofrecer tratamiento con morfina u otras sustancias a adictos en prisión, Anslinger refirió a experimentos similares en otras partes que, según él, solamente habían empeorado la situación de los presos. Ante una breve objeción de Siurob, Anslinger recordó que la ley estadounidense prohibía cualquier tipo de tratamiento similar y que ésa era la medida con la cual el comisionado iba a juzgar las políticas de otros países.

En caso de que Siurob lograra la suspensión de la reforma, Anslinger se ofreció a interceder ante el oAc para que México no fuera sancionado en próximas sesiones por incumplir los convenios internacionales, como él mismo lo había sugerido con anterioridad. Además, dio su palabra de levantar el embargo medicinal en el momento en que se suspendiera el reglamento.

Resulta sugerente -como ejemplo del manejo de los tiempos que hace la burocracia estadounidense con la información a su alcance-que durante la visita de Siurob a Estados Unidos en mayo, la Oficina de Aduanas decidiera no publicar ni liberar fotografías sobre los éxitos de la campaña de erradicación de opio celebrada en Sinaloa esa misma primavera. En su lógica, en el contexto de crisis por el que atrave- 
saba la relación, no podían permitirse publicitar al gobierno mexicano en ningún aspecto. ${ }^{86}$

Al enterarse Hans Morgenthau de lo acontecido en las negociaciones con Siurob, el entonces secretario del Tesoro felicitó a Anslinger y subrayó el gran triunfo que significaba no solamente que los mexicanos hubiesen recapacitado, sino que estuvieran dispuestos a desarrollar su política antidrogas bajo la tutoría del comisionado. ${ }^{87}$ Anslinger había hecho una apuesta arriesgada pero victoriosa; eventualmente sería retribuido por el War Production Board para coordinar el flujo de narcóticos hacia Estados Unidos, una posición que lo impulsó irremediablemente como actor clave de la política antidrogas de EE.UU. hasta la década de los años sesenta.

A su regreso a México, un transfigurado Siurob presentó al Consejo de Salubridad General el resultado de sus gestiones. Lo que antes eran consignas revolucionarias ahora eran "datos científicos y técnicos" por los cuales debía suspenderse el Reglamento. 88 "Lo único que da frutos óptimos", dijo, "era la relegación del toxicómano en granjas adecuadas a [tal] efecto". ${ }^{89}$ Algunos médicos reforzaron la posición de Siurob; el resto calló.

El tres de julio de 1940, el Dof publicó la suspensión "por tiempo indefinido" del Reglamento de Toxicomanías. ${ }^{90}$ Como pretexto, se alegó la dificultad de transportar drogas narcóticas de Europa a México en medio de la situación de guerra. La explicación -media verdad, media mentira, pero ciertamente verosímil para el gran público- ni siquiera provino del ingenio de Siurob, sino de una recomendación hecha por un

${ }^{86}$ Véase Thos J. Gorman a Supervising Customs Agent, 4 de mayo de 1940, RG 170, Caja 22, fólder 2, NARA.

${ }^{87}$ Hans Morgenthau a Harry J. Anslinger, 9 de mayo de 1940, RG 170, Caja 22, fólder 2, NARA.

88 “Acta de sesión, número 16", 11 de junio de 1940, Consejo de Salubridad General, Actas de Sesión, Libros, 2, AHSSA.

89 "Acta de sesión, número 16".

${ }^{90}$ Véase "Decreto que suspende la vigencia del Reglamento Federal de Toxicomanías", Diario Oficial de la Federación, 3 de julio de 1940. 
funcionario estadounidense semanas atrás. No se admitió que el reglamento contradijera la Convención de Ginebra o que EE.UU. hubiese presionado para una suspensión que, en la práctica, fue una derogación. Diez días después cerró el dispensario en la calle Sevilla. Tan pronto como pudo, el embajador Castillo Nájera escribió a Anslinger para comunicar sobre la revocación del reglamento y pedir que se levantara el embargo impuesto por el comisionado semanas atrás. ${ }^{91}$ Así se hizo. La Banda de música dejó de tocar.

\section{Conclusiones}

Este artículo buscó examinar el papel que desempeñaron distintas instituciones y actores del gobierno de EE.UU. en la suspensión del Reglamento de Toxicomanías de 1940, así como analizar la defensa que de éste hicieron funcionarios del DSP y de la SRE. Con este fin, y bajo una perspectiva que enfatiza la relevancia de las relaciones transgubernamentales -esto es, el conjunto de interacciones entre subunidades de diferentes gobiernos no controladas por los órganos centrales de los gobiernos nacionales- el artículo reconstruyó los detalles de la relación México-EE.uU. con respecto a este tema.

En lo que atañe a EE.uU., se demostró que, a pesar de la existencia de desacuerdos fundamentales en la forma de abordar el problema entre la embajada estadounidense y el comisionado Anslinger, estos desacuerdos no fueron identificados ni explotados por los mexicanos. Además, en lo esencial, hubo acuerdo en que sólo mediante la implementación de mecanismos de coerción iba a lograr detenerse el plan de Salazar.

En perspectiva, la decisión de embargar los medicamentos a México resulta, cuando menos, sorprendente. El embargo ocurrió en un momento en que los analistas de la relación

${ }^{91}$ F. Castillo Nájera a Harry J. Anslinger, 25 de julio de 1940, RG 170, Caja 22, fólder 2, NARA. 
México-EE.uU. no han dudado en etiquetar como uno de los pocos en que predominó la "cooperación" sobre el "conflicto". ${ }^{92}$ En un contexto de guerra y en el marco de la negociación de una alianza militar, los diplomáticos estadounidenses tensaron una liga que habría derivado en un problema diplomático mayor. ${ }^{93}$

Si la unidad de criterios y la disciplina fue lo que definió la posición estadounidense, lo opuesto debe decirse de la parte mexicana. El artículo presentó evidencia de la ausencia de coordinación de los diplomáticos y responsables de tomar decisiones en México, un tema sobre el que ya elaboró Isaac Campos. ${ }^{94}$

En varias ocasiones, incluyendo la plática informal del 28 de mayo de 1939 en Ginebra, así como en las reuniones de principios de mayo de 1940 en Washington D.C., los estadounidenses identificaron la falta de consenso en la posición mexicana. Por si fuera poco, contaron con improbables aliados que, aun con pequeñas acciones o (precisamente) por la ausencia de éstas, ayudaron a debilitar la defensa diplomática del Reglamento. Asimismo, el artículo presentó evidencia de que entre los funcionarios mexicanos predominaron los problemas de comunicación, así como el desconocimiento de los postulados centrales de la reforma del Reglamento. Todo ello evitó su defensa adecuada en foros internacionales, así como en mesas de negociación formales e informales.

El artículo contribuye al estudio de la relación MéxicoEstados Unidos en materia de narcóticos. Se trata de un epi-

92 Josefina Zoraida Vázquez y Lorenzo Meyer, México frente a Estados Unidos: un ensayo histórico, 1776-1980, México, El Colegio de México, 1982, p. 187; Susana Chacón, La relación entre México y los Estados Unidos, México, Fondo de Cultura Económica, 2008, p. 25.

${ }^{93}$ En los meses posteriores a la suspensión del Reglamento se firmó un acuerdo aeronáutico (abril de 1941), otro de cooperación antialemana (junio de 1941) y uno más que garantizaba la compra y venta de minerales estratégicos (julio de 1941). En enero de 1942 se creó la Comisión México-Norteamericana de Defensa Conjunta y unos meses después se concretó el Programa Bracero.

${ }^{94}$ Campos, art. cit. 
sodio que -de manera similar al cierre de fronteras, conocido como Operación Intercepción en 1969- ha sido revisado por la historiografía como un hecho que da cuenta de las condiciones de asimetría de ambos países y como ejemplo de la manera en que EE.uU. ha utilizado mecanismos de coerción para constreñir el margen de maniobra de México para ejecutar su política antinarcóticos. Esa perspectiva, sin embargo, necesita fundamentarse mejor. ¿Es el episodio sobre el Reglamento de Toxicomanías representativo de la dinámica bilateral? ¿Qué inferencias pueden generarse de este caso para el mejor examen de la relación México- EE.uU.? Son preguntas que quedan abiertas y sólo pueden responderse con mayor investigación documental. Hoy, cumplidos 80 años de la promulgación (y suspensión) del Reglamento de 1940, parece un buen momento para empezar.

\section{Archivos consultados}

Archivo General de la Nación, Agn, Ciudad de México, México.

National Archives and Records Administration, NARA, Maryland, Estados Unidos.

Archivo Histórico de la Secretaría de Salud y Asistencia, AHssa, Ciudad de México.

Archivo Histórico Genaro Estrada

\section{BIBLIOGRAFÍA SEGUNDARIA}

Astorga, Luis, El siglo de las drogas: el narcotráfico, del porfiriato al nuevo milenio, México, Grijalbo/Proceso, 2012.

"Campaña contra una lacra social”, El Universal (sección Editorial), 23 de mayo de 1940.

Campos, Isaac, "A Diplomatic Failure: The Mexican Role in the Demise of the 1940 Reglamento Federal de Toxicomanías", Third World Quarterly 39, núm. 3, 2017. 
Chacón, Susana. La relación entre México y los Estados Unidos. México, Fondo de Cultura Económica, 2008.

Daniels, Josephus, Shirt-Sleeve Diplomat, Chapel Hill, The University of North Carolina Press, 1947.

D’Artigues, Katia, "El presidente que legalizó las drogas", El Universal, el 10 de febrero de 2017.

Schievenini, José Domingo, "La criminalización del consumo de marihuana en México (1912-1961)", tesis de doctorado, UNAM, 2018.

Enciso, Froylán, "Los fracasos del chantaje: régimen de prohibición de drogas y narcotráfico”, en Mónica Serrano y Arturo Alvarado (eds.), Seguridad Nacional y Seguridad Internacional: Los grandes problemas de México, xv, pp. 62-104, México, D.F., El Colegio de México, 2010.

Flores Guevara, Mariana, "La alternativa mexicana al marco internacional de prohibición de drogas durante el cardenismo", tesis de licenciatura, México, El Colegio de México, 2013.

Motтier, Nicole, "Drug Gangs and Politics in Ciudad Juárez: 19281936”, Mexican Studies/Estudios Mexicanos 25, núm. 1, 2009, pp. 19-46.

Nye, J.S., y R.O. Keohane, "Transgovernmental Relations and International Organizations”, World Politics: A Quarterly Journal of International Relations, 1974, pp. 39-62.

Pérez MonTfort, Ricardo, Tolerancia y prohibición: aproximaciones a la historia social y cultural de las drogas en México (1840-1940), México, D.F., Debate, 2016.

"Decreto que suspende la vigencia del Reglamento Federal de Toxicomanías", Diario Oficial de la Federación, 3 de julio de 1940.

"Reglamento Federal de Toxicomanías del Departamento de Salubridad Pública (1940)", Diario Oficial de la Federación, 17 de febrero de 1940.

Salazar Viniegra, Leopoldo, "Opio y política: Historia de una humillación”, Excélsior, 19 de diciembre de 1945.

Salazar Viniegra, Leopoldo, "The Struggle against Toxicomanías", presentado en International Conference of Executives of the Law of the Pacific Coast, Ciudad de México, Bellas Artes, 1939 . 
Smith, Benjamin T., "The Dialectics of Dope: Leopoldo Salazar Viniegra, the Myth of Marijuana, and Mexico's State Drug Monopoly", en Susannah Wilson (ed.), Prohibitions and Psychoactive Substances in History, Culture and Theory, pp. 111-132, Londres, Routledge, 2019.

Smith, Nathaniel Lee, “'Cured of the Habit by Force': The United States and the Global Campaign to Punish Drug Consumers, 1898-1970", tesis de doctorado, Chapel Hill, The University of North Carolina, 2007.

VÁzquez, Josefina Zoraida y Lorenzo Meyer, México frente a Estados

Unidos: un ensayo histórico, 1776-1980, México, El Colegio de México, 1982.

WALKer III, William. "Control across the Border: The United States, Mexico, and Narcotics Policy, 1936-1940". Pacific Historical Review 47, núm. 1 (1978): 91-106. 